\title{
Temporal Logic Control of Switched Affine Systems with an Application in Fuel Balancing
}

\author{
Petter Nilsson, Necmiye Özay, Ufuk Topcu and Richard M. Murray
}

\begin{abstract}
We consider the problem of synthesizing hierarchical controllers for discrete-time switched affine systems subject to exogenous disturbances that guarantee that the trajectories of the system satisfy a high-level specification expressed as a linear temporal logic formula. Our method builds upon recent results on temporal logic planning and embedded controller synthesis. First, the control problem is lifted to a discrete level by constructing a finite transition system that abstracts the behavior of the underlying switched system. At the discrete level, we recast the problem as a two player temporal logic game by treating the environment driven switches as adversaries. The solution strategy for the game (i.e. the discrete plan) is then implemented at the continuous level by solving finite-horizon optimal control problems that establish reachability between discrete states and that compensate the effects of continuous disturbances. We also extend the earlier work by making efficient use of propositions in the temporal logic formula to drive the abstraction procedure and to facilitate the computation of continuous input at implementation time.

An aircraft fuel system example is formulated; and solved using the proposed method. This sample problem demonstrates the applicability of the abstraction procedure and correct-byconstruction controllers to regulate the fuel levels in multiple tanks during interesting operations like aerial refueling.
\end{abstract}

\section{INTRODUCTION}

Temporal logics provide a formal means to specify and verify the correct behavior of systems and have been extensively used in digital circuit design and software engineering [1], [2]. Due to their expressive power, there has been a recent interest in the use of temporal logics as specification languages for dynamical systems (see, for instance, [3], [4], [5], [6]). Leveraging recent results on temporal logic planning and embedded controller synthesis [7], [8], [9], in this paper we present a method for automatic synthesis of controllers for switched affine systems. The controllers are correct-by-construction in the sense that any execution of the system is guaranteed to fulfill a given linear temporal logic (LTL) specification.

In order to enable discrete planning we lift the problem to the discrete level, which requires computing a finite transition system. The ability to construct a controller depends on how many possible transitions that are established in this finite transition system. The first contribution of the present

This work was supported in part by Caltech Summer Undergraduate Research Fellowship, the FCRP consortium through the Multiscale Systems Center (MuSyC) and the Boeing Corporation.

P. Nilsson is with the Royal Institute of Technology (KTH), Sweden. email: pettni@kth.se

N. Ozay, U. Topcu and R. M. Murray are with Control and Dynamical Systems, California Institute of Technology, Pasadena, CA 91125, USA. emails: \{necmiye, utopcu, murray\} ecds.caltech.edu paper is to enhance the performance of the discretization method proposed by Wongpiromsarn et al. [7] by relaxing the requirements for allowed transitions between discrete states, while still ensuring system correctness. By allowing more transitions it is possible to synthesize controllers for a wider range of systems and at the same time get a system performance that is closer to optimal.

Our second contribution is the extension of the temporal logic planning framework to systems whose dynamical mode can be changed by the environment, so called switched systems. Control of switched systems is a challenging problem and has attracted considerable attention [10]. Although a thorough survey is beyond the scope of this paper, we give a very brief overview of existing work. The main body of results is on stability analysis for certain classes of switching [11], [12], [13]. Blanchini et al. [14] provide a characterization of all stabilizing controllers for linear switched systems under arbitrary switching. Tarraf et al. [15] use finite abstractions to design stabilizing switching controllers for second order systems. We also use finite abstractions, but instead of stability we consider controller design for an expressive subset of LTL specifications. Our framework can handle arbitrary switching. Moreover, it allows users to specify a priori assumptions on the switching sequence. For instance, it is possible to accommodate different time scales for the switch sequence and the system dynamics or to restrict certain transitions among different modes with an LTL formula if such a priori information is available.

Finally, motivated by design challenges in vehicle management systems (VMS) that control and coordinate a number of subsystems of aerial vehicles (e.g., flight controllers, electrical systems, environmental control systems, fuel systems, deicing units, and landing gear [16], [17], [18]), we apply the proposed method to a VMS example. In particular, we demonstrate how a switched system can be controlled with the proposed technique by synthesizing a controller for the fuel subsystem. The system is switched between normal operation, where fuel is consumed at a constant rate, and aerial refueling mode, where the net inflow of fuel to the system is positive. The goal is to balance the fuel volumes in different tanks and to ensure safe operation under all possible switching sequences in a prespecified class.

The rest of the paper is organized as follows. In section II, we introduce some definitions and the formal problem setting. Section III details the proposed hierarchical approach. We present the fuel balancing case study in section IV. Finally, section V concludes the paper with some remarks and directions for future research. 


\section{PRELIMINARIES AND PROBLEM FORMULATION}

This section contains some definitions and background information that will be used throughout the paper, as well as a formal definition of the problem considered.

\section{A. Polytopes}

A polytope $P$ is a convex set in $\mathbb{R}^{n}$, usually defined as the intersection of a finite number of half planes, i.e., $P=\left\{x \in \mathbb{R}^{n} \mid A x \leq b\right\}$. The projection of a polytope onto its first $m$ coordinates is the set $\operatorname{proj}_{m}(P)=\left\{x \in \mathbb{R}^{m} \mid \exists y \in\right.$ $\left.\mathbb{R}^{n-m}:\left[\begin{array}{ll}x & y\end{array}\right] \in P\right\}$. Several algorithms exist for computing the projection of polytopes, the optimal choice of algorithm depends on the characteristics of the polytope. In this paper polytopes are used to define cells in a continuous state space.

We say that two disjoint polytopes $P_{1}=\left\{x \in \mathbb{R}^{n} \mid A x \leq b\right\}$ and $P_{2}=\left\{x \in \mathbb{R}^{n} \mid C x \leq d\right\}$ are order 1 neighbors if the set $\left\{x \in \mathbb{R}^{n} \mid[A]_{i} x \leq[b]_{i}+\varepsilon\right\} \cap\left\{x \in \mathbb{R}^{n} \mid[C]_{j} x \leq[d]_{j}+\varepsilon\right\}$ is non-empty for some choice of inequalities $[A]_{i} x \leq[b]_{i}$ and $[C]_{j} x \leq[d]_{j}$ from the two polytopes, where $[A]_{i}$ denotes the $i^{t h}$ row of $\mathrm{A}$. We generalize the concept of neighbors by calling neighbors of neighbors order 2 neighbors, and so on.

\section{B. Environment Switched Systems}

We consider a switched system, consisting of a plant and its environment, where the plant has $N_{d}$ different dynamical modes it can switch among. We assume that each such mode $\mathcal{M}_{k}$ is described by an affine dynamical model of the following form:

$$
\mathcal{M}_{k}:\left\{\begin{array}{l}
s(t+1)=A_{k} s(t)+B_{k} u(t)+E_{k} d(t)+K_{k}, \\
u(t) \in U_{k}(s(t)) \\
d(t) \in D_{k},
\end{array}\right.
$$

where $U_{k}(s(t))$ and $D_{k}$ are polytopes containing possible values for input and disturbance, respectively. In particular, $u(t) \in U_{k}(s(t))$ constitutes inequality constraints linear in $u(t)$ and $s(t)$ that represents the possibility of having the allowable input values depend on the state. We denote the dimension of the state space by $n$ and the dimension of the input space by $m$. The vector $K_{k}$, the affine offset term, has dimension $n$ and describes constant changes in the state.

An environment switched system is a system where the dynamical mode is controlled by the environment (i.e., the switches are uncontrollable). Assume that the environment controls a variable $e_{d}$, and that the dynamics of the plant depend on the value of $e_{d}$ (i.e., we have $\mathcal{M}_{e_{d}}$ ).

\section{Linear Temporal Logic}

Linear Temporal Logic (LTL) is an extension of the classical logic by including temporal operators. Apart from the usual operators negation $(\neg)$, disjunction $(\vee)$, conjunction $(\wedge)$ and implication $(\rightarrow)$, it provides the possibility to write statements including the temporal operators next $(\bigcirc)$, always $(\square)$, eventually $(\diamond)$ and until ( $\mathcal{U}$ ). This makes it possible to write a wide range of requirements on the desired behavior of a system.
Definition 1: An atomic proposition is a statement on a system variable $v$ that has a unique truth value (True or False) for a given value of $v$.

Given a set $\Pi$ of atomic propositions, an LTL formula is defined inductively as follows: (i) any atomic proposition $p \in \Pi$ is an LTL formula; and (ii) given LTL formulas $\varphi$ and $\psi, \neg \varphi, \varphi \vee \psi, \bigcirc \varphi$ and $\varphi \mathcal{U} \psi$ are also LTL formulas. Formulas involving other operators can be derived from these basic ones.

We consider LTL specification for a system $\mathbb{S}$ consisting of a plant and its environment. The set of system variables in $\mathbb{S}$ are $V=S \cup E$, where $S$ is the set of variables controlled by the plant, i.e. the variables in $s(t)$ in (1), and $E$ is the set of variables controlled by the environment. The set of possible states in $\mathbb{S}$ is therefore $\operatorname{dom}(V)=\operatorname{dom}(S) \times \operatorname{dom}(E)$ and every state $v$ of the system can be written $v=(s, e)$ where $s \in \operatorname{dom}(S)$ and $e \in \operatorname{dom}(E)$. Here, $\operatorname{dom}(V)$ stands for the domain of $V$, i.e., the set of valuations of $V$.

An execution $\sigma$ of $\mathbb{S}$ is an infinite sequence of its states, i.e., $\sigma=(s(0), e(0)),(s(1), e(1)), \ldots$ LTL formulas are interpreted over state sequences. We say $\varphi$ is satisfied by $\mathbb{S}$ if $\varphi$ is true for all executions of $\mathbb{S}$. We refer the reader to [1] for exact semantics of LTL.

\section{Problem Formulation}

We consider a system $\mathbb{S}$ together with some specification $\varphi$ on the system, given in the form

$$
\varphi \doteq\left(\varphi_{e} \rightarrow \varphi_{s}\right)
$$

written in LTL. Generally speaking, some assumptions $\varphi_{e}$ on the environment should ensure that the requirements $\varphi_{s}$ on the plant states are satisfied. We denote by $\Pi$ the set of atomic propositions from $\varphi$ on the variables in $V$. In our setup, the propositions on the plant states $s(t)$ are in the form of linear inequality constraints that define convex polytopes corresponding to the regions of interest in the state space. Environment assumptions, for instance, can be used to define the a priori information on the possible switching sequences. Next, we give a formal definition of the problem.

Problem 1: Given a system $\mathbb{S}$ (consisting of an environment switched system (1) and a set of environment variables) and an LTL specification, $\varphi$, of the form (2), find a control input $u(t)$ such that $\mathbb{S}$ satisfies $\varphi$ (i.e., whenever the assumptions on the environment hold, the trajectories of (1) will satisfy the requirements).

\section{HIERARCHICAL APPROACH}

The automatic planner/controller synthesis proposed in [6], [7], [8], [19] relies on several steps. In this paper, we adopt a similar hierarchical approach. For the sake of completeness, we present the overall methodology, highlighting the contributions required for reducing conservatism and for extending the results to switched systems.

Since the planner synthesis requires a finite number of states, it is necessary to construct a finite transition system, which we will denote by $\mathbb{D}$. The finite transition system contains a partition of $\operatorname{dom}(S)$ and $\operatorname{dom}(E)$ into a finite 
number of equivalence classes (or cells) $\mathcal{S}$ and $\mathcal{E}$. The resulting finite partition is denoted by $\mathcal{V}=\mathcal{S} \times \mathcal{E}$. In the following we will call $s \in \operatorname{dom}(S)$ a continuous state for the plant and $\varsigma \in \mathcal{S}$ a discrete state.

First, we partition the continuous state space $\operatorname{dom}(S)$ into a coarse partition that is proposition preserving, and we let each cell in the partition represent a discrete state. The partition is said to be proposition preserving if, for any discrete state in the partition, exactly the same atomic propositions in $\Pi$ are true for all continuous states inside the discrete state. We also make sure that the cells in the partition are convex to be able to do convex optimization calculation of input signals, as explained in III-D. Any non-convex cell can be divided into several convex cells, and if the larger nonconvex cell is proposition preserving the smaller convex cells will also be so. We call this initial proposition preserving partition $\mathcal{P}$.

Secondly, we need the possible transitions in the finite transition system, for each dynamical mode $\mathcal{M}_{k}$. That is, we need $N_{d}$ lists of possible transitions between the discrete states in the partition $\mathcal{P}$. The transitions are established through the concept of reachability, which is developed in III-A. In general it will not be possible to establish enough reachability relations between discrete states in the initial proposition preserving partition $\mathcal{P}$, since it is too coarse. Therefore further discretization has to be done to obtain a finer partition. We present in III-B a procedure that refines the partition in order to establish reachability relations. The refined partition together with the lists of possible transitions is the finite transition system $\mathbb{D}$.

Finally we synthesize a discrete planner for $\mathbb{D}$ and implement the planner by using a continuous controller that moves the plant between the discrete states, as discussed in III-C and III-D, respectively. The overall controller has a feedback structure that takes into account both the current state $s(t)$ and the current environment dependent mode $k$ (which also depends on $t$ ).

\section{A. Reachability}

Assume that $\mathcal{S}=\left\{\varsigma_{0}, \varsigma_{1}, \ldots \varsigma_{n}\right\}$ is a partition of the continuous state space $\operatorname{dom}(S)$. We define the mapping $T_{\mathcal{S}}$ : $\operatorname{dom}(S) \rightarrow \mathcal{S}$ that takes a continuous state to its corresponding discrete state. The inverse mapping $T_{\mathcal{S}}^{-1}\left(\varsigma_{i}\right)$ describes the set of all continuous states that are inside the discrete state $\varsigma_{i}$.

We also assume that there exists another proposition preserving partition $\mathcal{P}=\left\{\rho_{0}, \rho_{1}, \ldots \rho_{m}\right\}$ of $\operatorname{dom}(S)$, such that $\mathcal{S}$ is a refinement of $\mathcal{P}$. In other words, for every $\varsigma_{i} \in \mathcal{S}$ there exists a $\rho_{j} \in \mathcal{P}$ such that $T_{\mathcal{S}}^{-1}\left(\varsigma_{i}\right) \subseteq T_{\mathcal{P}}^{-1}\left(\rho_{j}\right)$, where $T_{\mathcal{P}}: \operatorname{dom}(S) \rightarrow \mathcal{P}$ is defined in accordance with $T_{\mathcal{S}}$. The two partitions $\mathcal{S}$ and $\mathcal{P}$ may be equal.

Now we define the mapping $P_{\mathcal{S}}: \mathcal{S} \rightarrow \mathcal{P}$ that takes a discrete state to the cell in the initial proposition preserving partition from which it originates, i.e. $P_{\mathcal{S}}\left(\varsigma_{i}\right)=\rho_{j} \Leftrightarrow T_{\mathcal{S}}^{-1}\left(\varsigma_{i}\right) \subseteq$ $T_{\mathcal{P}}^{-1}\left(\rho_{j}\right)$. Then we can define our idea of reachability in a fixed horizon length $N$, with respect to some dynamical mode $\mathcal{M}_{k}$.
Definition 2: A discrete state $\varsigma_{j}$ is reachable from another discrete state $\varsigma_{i}$ in $N$ steps if, starting from any continuous state $s(0) \in T_{\mathcal{S}}^{-1}\left(\varsigma_{i}\right)$, there exists a control sequence $u(0), u(1), \ldots u(N-1)$ that takes the plant to some continuous state $s(N) \in T_{\mathcal{S}}^{-1}\left(\varsigma_{j}\right)$ in accordance with the system dynamics $\mathcal{M}_{k}$. This should be possible for all disturbance sequences $d(0), d(1), \ldots d(N-1) \in D_{k}^{N}$. Furthermore, we require that $u(t) \in U_{k}(s(t))$ for times $t \in\{0,1, \ldots N-1\}$, and that $s(t) \in$ $T_{\mathcal{P}}^{-1}\left(P_{\mathcal{S}}\left(\varsigma_{i}\right)\right)$ for times $t \in\{1,2, \ldots N-1\}$.

The last requirement in the definition makes sure that the trajectory of the plant remains inside the proposition preserving cell, which is required to ensure correctness when implementing a correct discrete plan. Roughly speaking, the same set of atomic propositions will be true during the whole trajectory from $\zeta_{i}$ to $\varsigma_{j}$, which lets the continuous implementation inherit correctness from the discrete plan.

It is worth emphasizing that our notion of reachability makes better use of proposition preserving partitions. In particular, we use a less restrictive definition of reachability than what has been used in [7], [19]. Instead of enforcing the plant to remain inside the initial cell, i.e $s(t) \in T_{\mathcal{S}}^{-1}\left(\varsigma_{j}\right)$, during a discrete transition, we use the looser constraint $s(t) \in T_{\mathcal{P}}^{-1}\left(P_{\mathcal{S}}\left(\varsigma_{j}\right)\right)$. A relaxed definition of reachability makes it possible to establish more reachability relations, something that is crucial in order to make the controller synthesis realizable.

The partition refinement technique in the next section relies on solving the following problem:

1) The reachability problem: Given an initial discrete state $\varsigma_{i}$ and a final discrete state $\varsigma_{j}$, find the set $S_{0} \subseteq \varsigma_{i}$ such that $\zeta_{j}$ is reachable from $S_{0}$.

2) Solving the reachability problem: By using the dynamics (1) and assuming that the dynamical mode stays constant up to time $t-1$, it can be shown that the plant state at time $t$ can be written as $s(t)=A_{k}^{t} s(0)+$ $\sum_{i=0}^{t-1}\left(A_{k}^{i} B_{k} u(t-1-i)+A_{k}^{i} E_{k} d(t-1-i)+A_{k}^{i} K_{k}\right)$ for matrices $A_{k}, B_{k}, E_{k}$ and $K_{k}$ corresponding to the dynamical mode $\mathcal{M}_{k}$. That is, the state at time $t$ depends on the dynamical mode, the initial state and all disturbances and input signals up to time $t-1$. From the definition of reachability, we can write down the following constraints for a transition from $\varsigma_{i}$ to $\varsigma_{j}$ in $N$ steps:

- $s(0) \in T_{\mathcal{S}}^{-1}\left(\varsigma_{i}\right)$,

- $s(t) \in T_{\mathcal{P}}^{-1}\left(P_{\mathcal{S}}\left(\varsigma_{i}\right)\right)$ for all $t \in\{1,2, \ldots N-1\}$,

- $s(N) \in T_{\mathcal{S}}^{-1}\left(\varsigma_{j}\right)$,

- $u(t) \in U_{k}(s(t))$ for all $t \in\{0,1, \ldots N-1\}$.

We assume that $T_{\mathcal{S}}^{-1}\left(\varsigma_{i}\right), T_{\mathcal{P}}^{-1}\left(P_{\mathcal{S}}\left(\varsigma_{i}\right)\right), T_{\mathcal{S}}^{-1}\left(\varsigma_{j}\right)$ and $U_{k}(s(t))$ are all polytopes, i.e. there exists $L_{0}, L_{t}, L_{N}, L_{U}$ and $M_{0}, M_{t}, M_{N}, M_{U}$ such that for example $T_{\mathcal{S}}^{-1}\left(\varsigma_{i}\right)=\{x \in$ $\left.\mathbb{R}^{n} \mid L_{0} x \leq M_{0}\right\}$. By expressing the states as functions of previous inputs and disturbances, it turns out that all the constraints above can be stacked and expressed as a system of inequalities on the form

$$
L\left[s(0)^{T} \quad u(0)^{T} \quad u(1)^{T} \quad \ldots \quad u(N-1)^{T}\right]^{T} \leq M-\hat{d},
$$

a set that defines a polytope of dimension $n+N m$. This polytope contains all combinations of initial states and input 
sequences for which the reachability problem is feasible. The matrix $L$ is built from $A_{k}, B_{k}, L_{0}, L_{t}, L_{N}$ and $L_{U}$ and the vector $M$ from $M_{0}, M_{t}, M_{N}$ and $M_{U}$. An element $[\hat{d}]_{i}$ in the vector $\hat{d}$ represents, for the corresponding inequality in the polytope, the effect of the worst possible disturbance sequence for that inequality. Namely, the $i^{t h}$ element of $\hat{d}$ can be expressed as

$$
[\hat{d}]_{i}=\max _{d \in D_{k}^{N}}[G]_{i} d
$$

since this leads to the tightest constraints in the inequality (3); thus ensuring that the reachability relation holds for all possible disturbances. The matrix $\mathrm{G}$, representing the effect of disturbances, is built from $A_{k}, E_{k}, L_{0}, L_{t}, L_{N}$ and $L_{U}$ in a similar way as $L$ and $M$. By using the theory of polyhedral convexity it can furthermore be shown that only the extreme points $\bar{D}_{k}^{N}$ of the polytope $D_{k}^{N}$ have to be considered in the maximization, which leaves us with a finite number of points to check in order to find $\hat{d}$.

We want to calculate the set $S_{0}$ of possible starting points for which a valid control sequence $\hat{u}=u(0), u(1), \ldots u(N-1)$ exists. This leads to the following solution of the reachability problem, which is on the form of a projected polytope.

Proposition 1: Suppose $L, M, G$ and $\hat{d}$ are as above, and that $S_{0}$ is the projection of the polytope $\left\{x \in \mathbb{R}^{n+N m} \mid L x \leq\right.$ $M-\hat{d}\}$ onto its first $\mathrm{n}$ coordinates, i.e,

$$
S_{0}=\left\{s \in \mathbb{R}^{n} \mid \exists \hat{u} \in \mathbb{R}^{N m} \text { s.t. } L\left[\begin{array}{l}
s \\
\hat{u}
\end{array}\right] \leq M-\hat{d}\right\} .
$$

Then, the reachability problem is feasible for any $s(0) \in S_{0}$.

To calculate the initial set $S_{0}$, we use an iterative idea that comes from [20]. In the procedure, for which the pseudo code is given in Algorithm 1, the feasible initial set $S_{0}$ is computed by back-propagating the final set $\varsigma_{j}$ one time step at a time.

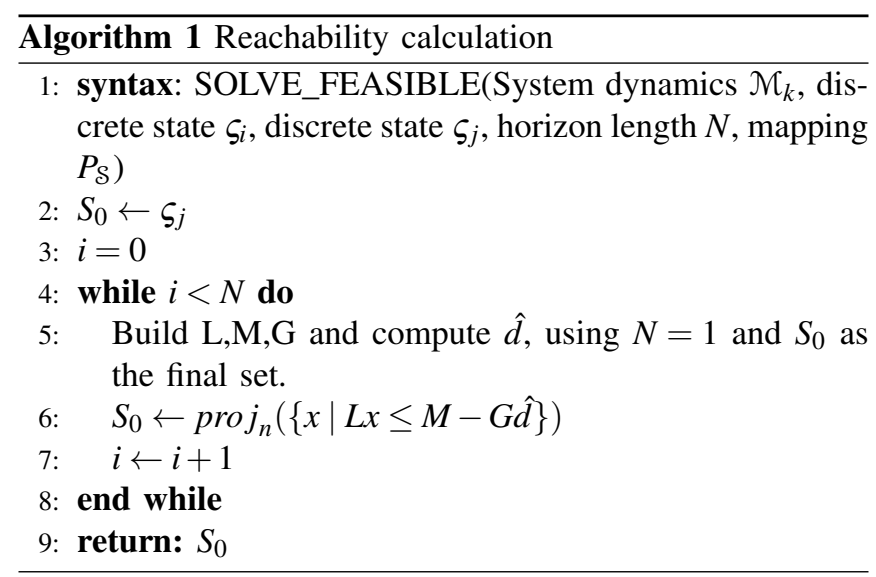

The advantage of this iterative approach lies in how it deals with disturbances. Instead of calculating the whole sequence of $N$ input signals at the same time, we recalculate the input in each time step and can thus take into account additional information, namely all disturbances up to the current time step in the iteration. When implementing a controller, it is accordingly also necessary to recompute the input signal at every time step, after measuring the latest disturbance. For each call to Algorithm 1, N projections of a polytope of dimension $n+m$ are computed.

Remark 1: We assume the mode remains constant in $N$ steps, which is the fixed time horizon of the abstraction. Hence, $N$ should be chosen by taking into account the time scale of the changes in the environment variable $e_{d}$, controlling the switches, and can be set to 1 for arbitrarily fast switching. Choosing $N>1$ requires additional synchronization assumptions (i.e., $e_{d}$ only changes at times $t=c N$ for integer $c$ ). In the context of VMS, environment for a subsystem is typically the other subsystems [17]. Hence, in VMS applications it is possible to achieve synchronization for $N>1$, either by using synchronous architectures or through communication.

\section{B. Specification Guided State Space Discretization}

To be able to establish reachability relations between the discrete states, in general the state space has to be partitioned further. We do this at the same time as we look for reachability relations. If it turns out after solving the reachability problem for a pair of cells that the final cell is reachable from only a part of the initial cell, the idea is to divide the initial cell into one feasible and one non-feasible part. This discretization algorithm can be found in Appendix I.

We do additional discretization separately for each dynamical mode $\mathcal{M}_{k}$ to obtain $N_{d}$ different partitions of the original continuous state space. Then we merge these partitions into one final single partition $\mathcal{S}$ which will have the reachability characteristics of all $N_{d}$ partitions. The merging procedure is given in Algorithm 2 and is essentially making new discrete states out of all possible intersections. Finally we recompute the possible transitions for this new partition $\mathcal{S}$ using each dynamical mode. This has to be done since the reachability relations may not be preserved when cells are divided. We denote the set of possible transitions for mode $k$ by the transition relation $R_{k} \subseteq \mathcal{S} \times \mathcal{S}$. In particular, for $\varsigma_{i}, \varsigma_{j} \in \mathcal{S},\left(\varsigma_{i}, \varsigma_{j}\right) \in R_{k}$ if $\varsigma_{j}$ is reachable from $\varsigma_{i}$ in the sense of Definition 2. The partition $\mathcal{S}$ together with the $N_{d}$ transition relations $R_{k}, k \in\left\{1, \ldots, N_{d}\right\}$, is our finite transition system D.

Proposition 2: Given that each of $\mathcal{S}_{1}, \mathcal{S}_{2}, \ldots, \mathcal{S}_{N_{d}}$ are partitions of $\operatorname{dom}(S)$, then, at each iteration of Algorithm 2, $\mathcal{S}$ will also be a partition of $\operatorname{dom}(S)$.

Proof: If $\mathcal{S}$ and $\mathcal{S}_{k}$ are two partitions of the same space $\operatorname{dom}(S)$, every point in $\operatorname{dom}(S)$ will lie in one and only one state $\varsigma_{i} \in \mathcal{S}$ and one and only one state $\varsigma_{j} \in \mathcal{S}_{k}$. Therefore $\left\{\varsigma_{i} \cap \varsigma_{j} \mid \varsigma_{i} \in \mathcal{S}, \varsigma_{j} \in \mathcal{S}_{k}\right\}$ is also a partition of $\operatorname{dom}(S)$. The result follows by induction.

\section{Planner Synthesis}

In this section, we discuss how to synthesize a discrete plan, given the finite transition system $\mathbb{D}$ constructed in the previous section and a temporal logic specification of the assume-guarantee form (2). We first recast the problem as a two player temporal logic game by treating the environment driven switches as adversaries. This reformulation allows us 


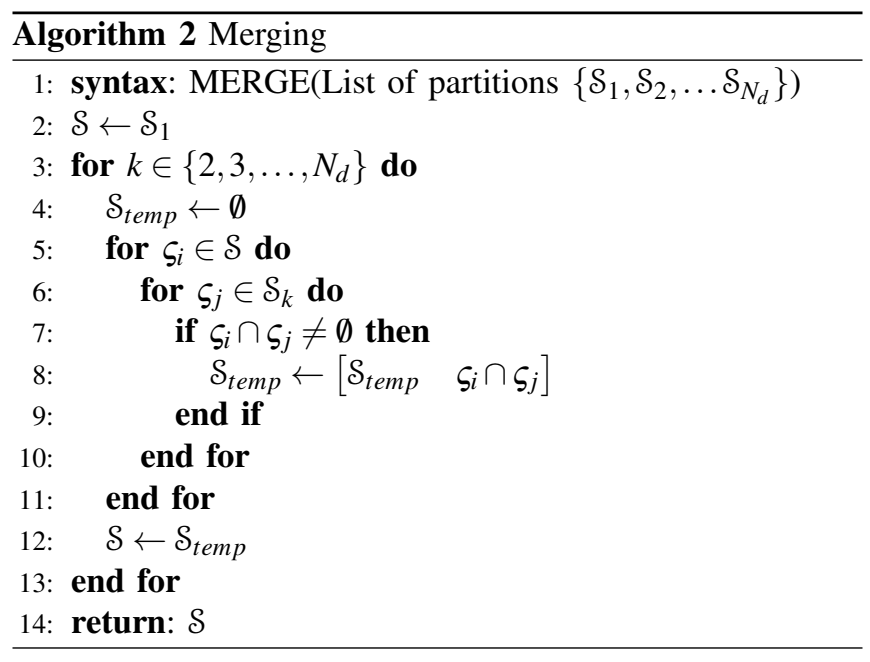

to employ a method due to Piterman et al. [9], [21] for synthesis of reactive discrete controllers in the presence of adversarial environment. Synthesis of reactive controllers for a general LTL formula has prohibitive complexity. However, as shown in [21], for an expressive subset of LTL, the socalled Generalized Reactivity(1) (GR(1)) formulas, synthesis can be achieved in time quadratic in the state space size (i.e., $|\mathcal{V}|$ ). We restrict ourselves to GR(1) formulas in this paper. Next, we give a very brief overview of the setup in [9], [21] and show how it relates to the problem we consider in this paper.

The main idea is to pose the planner synthesis problem as a two player game between the discrete states $\mathcal{S}$ in $\mathbb{D}$ and the set of environment variables, $\mathcal{E}$, that determine the dynamical mode. Consider the following GR(1) specification:

$$
\varphi \doteq\left(\varphi_{e} \rightarrow \varphi_{s}\right)
$$

where for $\alpha \in\{e, s\}$ both $\varphi_{\alpha}$ have the following structure :

$$
\varphi_{\alpha} \doteq \theta_{i n i t}^{\alpha} \wedge \bigwedge_{i \in I_{t_{\alpha}}} \square \psi_{i}^{\alpha} \wedge \bigwedge_{i \in I_{g \alpha}} \square \diamond J_{i}^{\alpha}
$$

In Eq. (5), $\varphi_{e}$ characterizes the assumptions on the environment and $\varphi_{s}$ describes the correct behavior of the system. In particular, $\theta_{\text {init }}^{\alpha}$ is an atomic proposition characterizing the initial conditions; $\psi_{i}^{\alpha}$ are atomic propositions characterizing invariants that should always be satisfied (e.g. safety requirements); and $J_{i}^{\alpha}$ are atomic propositions characterizing states that should be attained infinitely often. We assume (5) does not contain the next operator $(\bigcirc)$, hence it is stutter invariant. This technical assumption can be relaxed for the case when $N=1$. From (5) and $\mathbb{D}$ we construct an additional specification that describes all allowable moves:

$$
\varphi_{d} \doteq\left(\varphi_{e} \rightarrow \varphi_{s} \wedge \varphi_{\mathbb{D}}\right),
$$

where $\varphi_{\mathbb{D}}$ encodes the transition system $\mathbb{D}$. In particular, $\varphi_{\mathbb{D}} \doteq \bigwedge_{k=1}^{N_{d}} \bigwedge_{\varsigma_{i} \in \mathcal{S}} \square\left(\left(e_{d}=k \wedge s \in \varsigma_{i}\right) \rightarrow \bigcirc \bigvee_{\left(\varsigma_{i}, \varsigma_{j}\right) \in R_{k}}\left(s \in \varsigma_{j}\right)\right)$ Since we abstract the discrete model with fixed time horizon $N$, next operator $(\bigcirc)$ in (6), corresponds to $N$ steps later for the continuous dynamics and by construction, the propositions on $s$ remain unchanged during these $N$ steps. Note that if (5) is in GR(1) so is (6). Hence, the synthesis method proposed in [9], which is implemented in JTLV [22], can be used. If there exists a discrete plan that satisfies (6), we say the specification (or the synthesis problem) is realizable. In this case, the output of the synthesis algorithm is a partial function $f:(e(t), \varsigma(t), e(t+N)) \mapsto \varsigma(t+N)$ (possibly with some finite memory variable as an additional input argument that we ignore for simplicity of notation), represented by a finite state automaton, that maps the previous discrete system state $v=(\varsigma, e)$ and the current environment value $e$ to the next discrete plant state in the partition. We call $f$ the planner for the system.

Remark 2: We gave this simpler description for constructing $\varphi_{d}$ in (6) but, strictly speaking, one needs to be careful about causality unless $e(t+N)$ can be predicted at time $t$. In particular, GR(1) synthesis [9] assumes that the plant can react to the environment changes instantaneously, however we have $N$ step delay due to discretization. To ensure causality (i.e., to synthesize a planner $\tilde{f}:(e(t-N), \varsigma(t), e(t)) \mapsto$ $\varsigma(t+N))$, it is possible to define $\tilde{e}(t) \doteq e(t-N)$ and construct a specification $\tilde{\varphi}_{d}$ such that, under some mild technical assumptions, it leads to a GR(1) game between $\tilde{e}$ and $s$. The main idea is to replace the appearances of $e$ with $\bigcirc \tilde{e}$ in each atomic proposition of $\varphi_{d}$ that contains variables from both $\mathcal{S}$ and $\mathcal{E}$; and with $\tilde{e}$ elsewhere. All the examples in this paper work with this modification, however for the sake of simplicity, we skip the details of the construction.

\section{Implementation and Correctness of the System}

The states $\mathcal{S}$ in the finite transition system $\mathbb{D}$ form a subpartition of the initial proposition preserving partition $\mathcal{P}$ and thus $\mathcal{S}$ is also proposition preserving. A discrete planner can be synthesized using JTLV [22] by giving $\mathbb{D}$ and the corresponding set of atomic propositions for each discrete state as input, as described in III-C.

During execution, the planner will control between which discrete states the plant should move in order to fulfill the system specifications. In order to perform these discrete transitions, we need a continuous controller. We do this with a finite-horizon linear quadratic regulator (LQR), using the (convex) constraints of reachability in III-A. The cost function can be chosen to penalize state deviations and/or input. Like with the reachability algorithm, the final set must be propagated backwards in time to find the feasible set for each time step. In order to move from $\zeta_{i}$ to $\varsigma_{j}$ we calculate an input sequence $u^{*}$ by solving an optimization problem for a given initial position $s(0) \in T_{\mathcal{S}}^{-1}\left(\varsigma_{i}\right)$,

$$
u^{*}= \begin{cases}\arg _{\{u(t)\}_{0}^{N-1}} & \sum_{t=1}^{N}\left(s(t)^{T} Q_{t} s(t)+q_{t}^{T} s(t)\right)+ \\ & \sum_{t=0}^{N-1}\left(u(t)^{T} R_{t} u(t)\right), \\ \text { such that } & s(N) \in T_{\mathcal{S}}^{-1}\left(\varsigma_{j}\right), \\ & s(1) \in S_{0_{N-1}}, \\ & s(t) \in T_{\mathcal{P}}^{-1}\left(P_{\mathcal{S}}\left(\varsigma_{i}\right)\right), \\ & u(t) \in U(s(t)), \\ & \forall t \in\{1,2, \ldots, N-1\},\end{cases}
$$


where $S_{0_{N-1}} \subseteq T_{\mathcal{P}}^{-1}\left(P_{\mathcal{S}}\left(\varsigma_{i}\right)\right)$ is the set calculated by Algorithm 1 for which the reachability problem is feasible in $N-1$ steps. Only the first input signal $u(0)$ is used at each time step. When the plant has moved one step the new disturbance is taken into account to compute a new input signal, again using (7) but with the horizon length $N$ decreased by 1 .

By allowing the plant to move inside the whole original proposition preserving cell $T_{\mathcal{P}}^{-1}\left(P_{\mathcal{S}}\left(\varsigma_{i}\right)\right)$ during the transition, rather than enforcing it to stay inside $T_{\mathcal{S}}^{-1}\left(\varsigma_{i}\right)$ like has been done in earlier work, we optimize over a larger set of trajectories. Therefore the cost of the optimal trajectory for this relaxed problem is at least as low as before and in general lower.

Using this method the following statement can be made about the system behavior.

Proposition 3: Under the assumption that the switches only occur at times $t=c N$ for integer $c$, using the planner $f$ synthesized for (6) together with the controller (7) guarantees that the specification $\varphi$ in (5) is satisfied by the system.

Proof: The result follows from Proposition 1 in [6] together with stutter invariance assumption on $\varphi$ and the fact that we require that the trajectory of the transitions between two discrete states should lie inside the parent cell $T_{\mathcal{P}}^{-1}\left(P_{\mathcal{S}}\left(\varsigma_{i}\right)\right)$ in the original proposition preserving partition. Hence, the same set of propositions are guaranteed to be satisfied during the transitions. Therefore the planner/controller system will behave correctly with respect to the specification $\varphi$ in (5).

\section{EXAMPLES}

The proposed strategy for automatic controller synthesis is illustrated with an academic example and an application in aircraft fuel system. To build the controllers we use The Temporal Logic Planning (TuLiP) Toolbox [23], a software package that is designed to synthesize controllers using the presented method, including an interface to JTLV.

\section{A. Comparison with Earlier Work on a Simple Example}

To illustrate the advantage of the proposed notion of reachability, we compare our approach with that in [7]. Since the latter cannot handle switched systems, we use a simple two-dimensional linear system with the following dynamics:

$$
\begin{array}{r}
{\left[\begin{array}{l}
s_{1}(t+1) \\
s_{2}(t+1)
\end{array}\right]=\left[\begin{array}{l}
s_{1}(t) \\
s_{2}(t)
\end{array}\right]+\left[\begin{array}{l}
u_{1}(t) \\
u_{2}(t)
\end{array}\right]+\left[\begin{array}{l}
d_{1}(t) \\
d_{2}(t)
\end{array}\right],} \\
\left|u_{1}(t)+u_{2}(t)\right| \leq 0.16 \\
\left|d_{1}(t)\right|,\left|d_{2}(t)\right| \leq 0.04
\end{array}
$$

The state space is the set $\left\{[x y] \in \mathbb{R}^{2} \mid 0 \leq x \leq 3,0 \leq y \leq 2\right\}$ and the initial proposition preserving partition consists of 6 identical square cells. We discretize the state space with Algorithm 3 in Appendix I, using the notion of reachability in Definition 2 and the corresponding definition from [7], and keeping the rest of the parameters the same (i.e., $N=8$ and min_vol $=0.1)$. The specifications on the system require the plant to be able to move between the lower left and the upper right cells in the initial partition. It turns out that with this set-up, discretizing using the reachability constraints in [7]

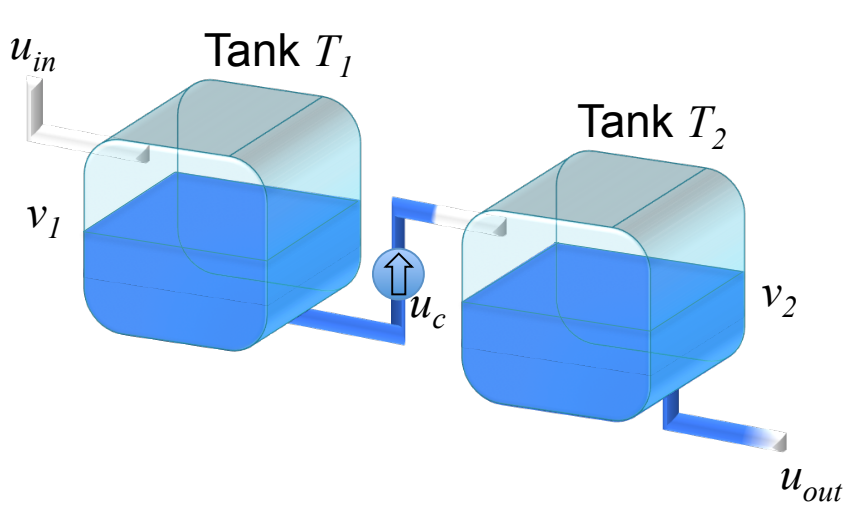

Fig. 1. Fuel tank system.

results in 29 possible transitions (including self transitions) between a total of 23 discrete states, which in this case is not enough to make the specification realizable. However, using the new relaxed constraints, a total of 34 discrete states are created with 93 transitions between them, which makes the controller synthesis realizable.

\section{B. Application in Fuel Balancing}

In this example we consider a switched affine system and illustrate the application of overall framework. The dynamical system we look at consists of two fuel tanks $T_{1}$ and $T_{2}$ in an airplane and the state variables are the two fuel volumes $v_{1}$ and $v_{2}$ in these tanks. We suppose that the maximum capacity in both tanks is 10 fuel units. The system can be controlled by moving fuel from tank $T_{1}$ to tank $T_{2}$ by using a pump, at a maximum rate of 3 fuel units per time step. This problem has only one control signal but two state variables, so it is not fully actuated. Such systems are often hard to control.

We model the system with two dynamical modes, one for normal operation and one for aerial refueling mode. During aerial refueling mode a tank plane is flying next to our plane and fills up fuel in tank $T_{1}$ at a rate of 3 fuel units per time step. We assume that the plane consumes 1 fuel unit per time step and that this fuel is taken from tank $T_{2}$. This gives the following model for the dynamics:

$$
\left[\begin{array}{l}
v_{1}(t+1) \\
v_{2}(t+1)
\end{array}\right]=\left[\begin{array}{ll}
1 & 0 \\
0 & 1
\end{array}\right]\left[\begin{array}{l}
v_{1}(t) \\
v_{2}(t)
\end{array}\right]+\left[\begin{array}{c}
-1 \\
1
\end{array}\right] u_{c}(t)+\left[\begin{array}{c}
u_{i n}(t) \\
-1
\end{array}\right],
$$

where $u_{\text {in }}(t)$ can be either 0 or 3 , depending on the dynamical mode. The restriction on the input signal can be written on polytope form as

$$
\left[\begin{array}{cc}
1 & 0 \\
-1 & 0 \\
1 & -1
\end{array}\right]\left[\begin{array}{l}
u_{c}(t) \\
v_{1}(t)
\end{array}\right] \leq\left[\begin{array}{c}
3 \\
0 \\
u_{\text {in }}(t)
\end{array}\right],
$$

where the last inequality makes sure that not more fuel than what is available can be moved from tank $T_{1}$.

1) Specifications: What we want to achieve with this system is to keep the difference between the two fuel levels low. The motivation for this is that a big difference may cause instability in the airplane. It is assumed that the refueling 


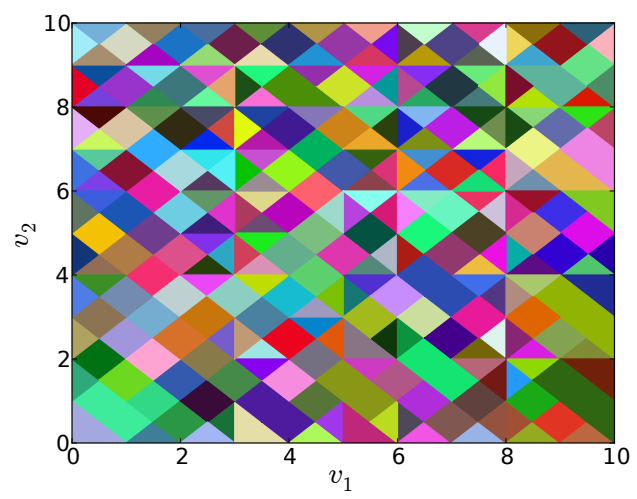

Fig. 2. Final state space partition, each colored area represents a discrete state.

mode will be initiated when the levels are too low, so that the system will never run out of fuel completely, and that the refueling mode will stop when tank $T_{2}$ is almost full. We therefore give the following requirements and specifications on the system.

\section{Assumptions:}

- When $v_{1}+v_{2} \leq 2$ and $u_{\text {in }}=0$, next step require $u_{\text {in }}=3$.

- When $v_{2} \geq 8$ and $u_{\text {in }}=3$, next step require $u_{\text {in }}=0$.

\section{Requirements:}

- Always require that $\left|v_{1}-v_{2}\right| \leq 2$.

- Always eventually require that $\left|v_{1}-v_{2}\right| \leq 1$.

The first requirement ensures safe operation, while the second requirement makes sure that improved performance is achieved infinitely often.

2) State Space Discretization: We run the discretization algorithm in Appendix I two times to obtain one partition for each dynamical mode, using time step $N=1$ for reachability in one time step (allowing for the system to switch between dynamical modes at every time step). Then we merge the two partitions using Algorithm 2. The resulting final partition of the state space is shown in Fig. 2. Finally, we make repeated calls to Algorithm 1 in order to get two lists of possible transitions between discrete states in this partition, one list for each dynamical mode. The partition and the possible transitions can now be plugged into JTLV to synthesize a discrete planner, and it turns out that the specifications are realizable for this particular problem. The discrete states that fulfill the specification are the states that lie at most 2 length units away from the line $v_{1}=v_{2}$. Since the planner synthesis is successful, we have a way of controlling the system to always stay inside this area.

For this example, the discretization procedure took 282 seconds on a MacBook Air $1.86 \mathrm{GHz}$. A planner for the resulting 277 discrete states could be synthesized in 15 seconds on the same computer.

3) Results: The planner is implemented in a simulation together with a controller on the form of (7). We put a weight in the optimization that punishes deviations from the centers of the cells. The simulation is run in 25 time steps and the result is shown in Fig. 3. As can be seen,
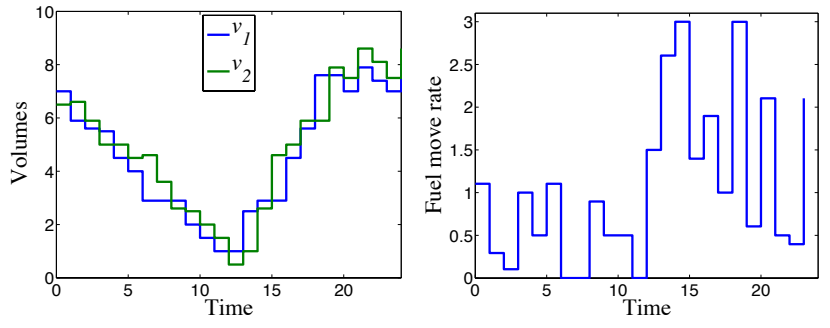

Fig. 3. Simulation results. Left: Tank levels. Right: Fuel move rate, $u_{c}(t)$

the two levels of fuel track each other closely, fulfilling the system specification. In the beginning of the experiment the plane is in normal operation mode, which can be seen by the declining fuel levels. When the fuel levels become low the system is switched to aerial refueling mode and fuel starts to be added to tank $T_{1}$, causing both levels to increase. And we allow some arbitrary switching consistent with environment assumptions in the last 5 time steps. Fig. 3 also shows how much fuel is being moved from tank $T_{1}$ to tank $T_{2}$ during the simulation.

\section{CONCLUSIONS AND FUTURE WORKS}

We have presented a strategy for automatic synthesis of a planner and a controller for a switched system that is correct by construction, in the sense that once synthesized, the planner/controller is guaranteed to fulfill the specifications. The strategy can be implemented on systems that are switched, i.e. whose dynamical mode is controlled by the environment. Most of the results on switched systems are either only sufficient conditions or computationally expensive; and the proposed method is no exception. However, as shown in the paper, controller synthesis for switched systems from temporal logic specifications, can be recast as a two-player game by treating the switching sequence as environment. This reformulation has enabled using tools from reactive controller synthesis for linear or piecewise affine systems with appropriate modifications. An important step in the procedure is to partition the continuous state space into a finite number of discrete states, in order to enable planning. We have shown improved performance in discretization step by effectively using propositions in the temporal logic formula while computing reachability relations.

The planner/controller synthesis strategy has been implemented on an example with fuel tanks. The fuel tank system is under-actuated and is switched by the environment between two dynamical modes. A planner could successfully be synthesized and therefore any execution is guaranteed to fulfill the specification.

Future work includes tuning the discretization procedure for switched systems by taking into account the a priori information on the switches to reduce potential conservatism. We are also interested in investigating the convergence properties of the approach in the sense that as finer and finer partitions are considered whether it would be possible to obtain a control strategy, that satisfies the specification, at discrete level whenever one exists for the continuous system. 
APPENDIX I

\section{DISCRETIZATION ALGORITHM}

This appendix summarizes a discretization algorithm proposed in [6], [7], [19] and also used in this paper. The purpose of this algorithm is to refine a given partition of the state space and at the same time establish reachability relations between discrete states in the partition.

An iteration of the proposed algorithm starts with picking two arbitrary states $\varsigma_{i}$ and $\zeta_{j}$ in the current partition. Then the subset $S_{0} \subseteq \varsigma_{i}$ from which $\varsigma_{j}$ is reachable is computed. If $S_{0}$ is found to be non-empty, but not the same as $\varsigma_{i}, \varsigma_{i}$ is partitioned into $\varsigma_{i} \cap S_{0}$ and $\varsigma_{i} \backslash S_{0}$, and thus the number of discrete states increases by 1 . By construction the state $\varsigma_{j}$ will be reachable from the new state $\varsigma_{i} \cap S_{0}$. The idea is to keep doing this until enough transitions have been established inside the state space. The pseudo-code of this algorithm is given in Algorithm 3, where a minimum cell volume is used as a termination criteria. To improve the speed of the algorithm, it can be restricted it to check for transitions between states that are neighbors of some order $N_{N B}$, using the notation introduced in II-A.

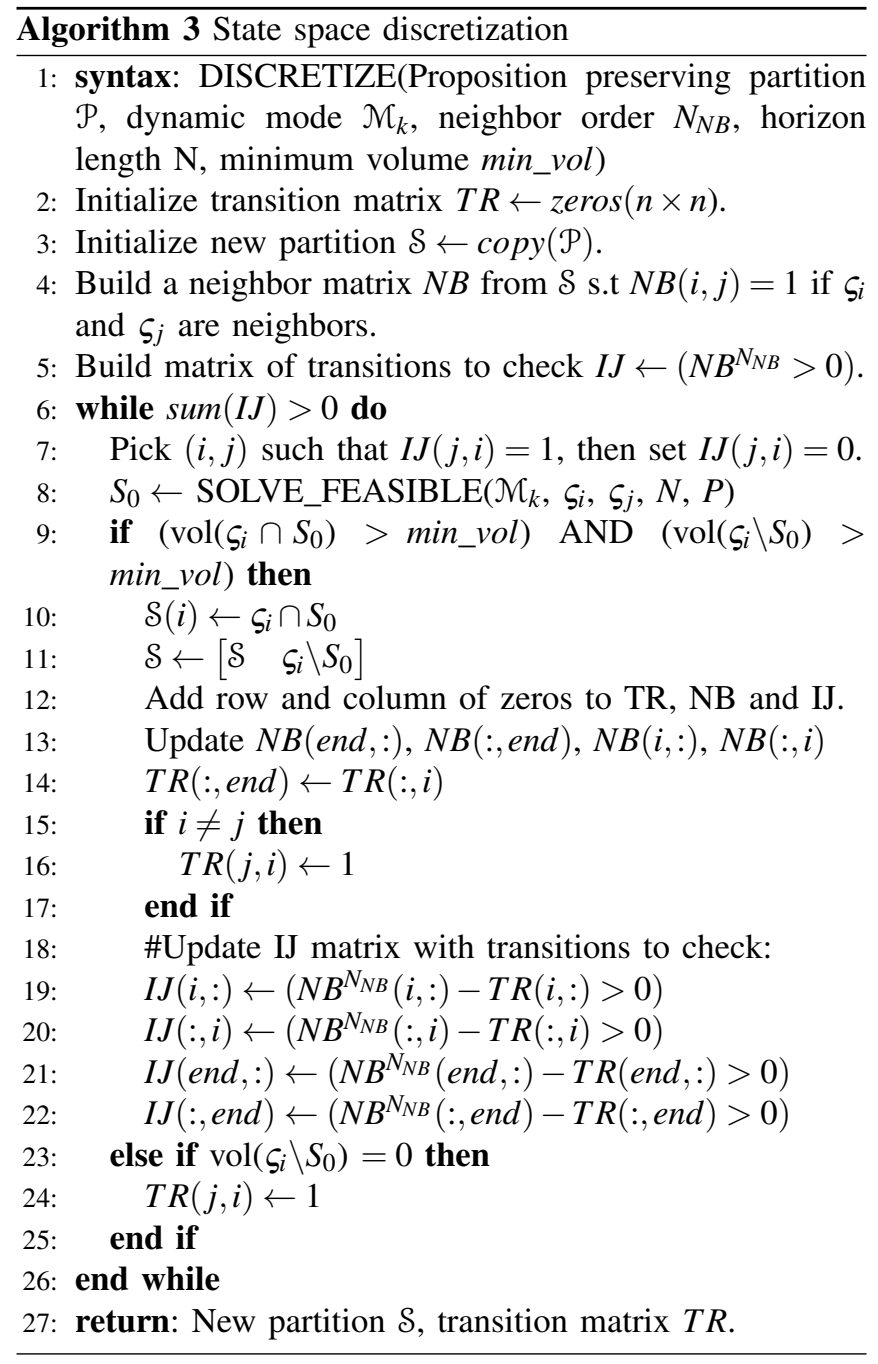

\section{REFERENCES}

[1] Z. Manna and A. Pnueli, The temporal logic of reactive and concurrent systems. Springer-Verlag, 1992.

[2] A. Pnueli, "Applications of temporal logic to the specification and verification of reactive systems: a survey of current trends," Current Trends in Concurrency. Overviews and Tutorials, pp. 510-584, 1986.

[3] P. Tabuada and G. J. Pappas, "Linear time logic control of linear systems," IEEE Trans. on Automat. Contr., vol. 51, no. 12, pp. 1862$1877,2006$.

[4] M. Kloetzer and C. Belta, "A fully automated framework for control of linear systems from temporal logic specifications," IEEE Trans. on Automat. Contr., vol. 53, no. 1, pp. 287-297, 2008.

[5] G. E. Fainekos, A. Girard, H. Kress-Gazit, and G. J. Pappas, "Temporal logic motion planning for dynamic robots," Automatica, vol. 45, no. 2 , pp. 343-352, 2009.

[6] T. Wongpiromsarn, U. Topcu, and R. M. Murray, "Receding horizon temporal logic planning for dynamical systems," in Proc. IEEE CDC, Shanghai, China, Dec. 2009.

[7] _ - "Automatic synthesis of robust embedded control software," in AAAI Spring Symposium on Embedded Reasoning: Intelligence in Embedded Systems, 2010, pp. 104-111.

[8] _ "Receding horizon control for temporal logic specifications," in HSCC, 2010, pp. 101-110.

[9] N. Piterman, A. Pnueli, and Y. Sa'ar, "Synthesis of reactive(1) designs," in VMCAI, 2006, pp. 364-380.

[10] D. Liberzon, Switching in systems and control, ser. Systems \& control. Birkhäuser, 2003.

[11] M. S. Branicky, "Multiple lyapunov functions and other analysis tools for switched and hybrid systems," IEEE Trans. on Automat. Contr. vol. 43, no. 4, pp. $475-482$, apr 1998.

[12] J. P. Hespanha, "Uniform stability of switched linear systems: Extensions of LaSalle's invariance principle," IEEE Trans. on Automat. Contr., vol. 49, no. 4, pp. 470-482, Apr. 2004.

[13] P. A. Parrilo and A. Jadbabaie, "Approximation of the joint spectral radius using sum of squares," Linear Algebra and its Applications, vol. 428, no. 10, pp. 2385 - 2402, 2008, special Issue on the Joint Spectral Radius: Theory, Methods and Applications.

[14] F. Blanchini, S. Miani, and F. Mesquine, "A separation principle for linear switching systems and parametrization of all stabilizing controllers," IEEE Trans. on Automat. Contr., vol. 54, no. 2, pp. 279 -292 , feb. 2009.

[15] D. C. Tarraf, A. Megretski, and M. A. Dahleh, "Finite approximations of switched homogeneous systems for controller synthesis," IEEE Trans. on Automat. Contr., vol. 56, no. 5, pp. 1140 -1145, may 2011.

[16] I. Moir and A. Seabridge, Aircraft Systems: Mechanical, Electrical, and Avionics Subsystems Integration. AIAA Education Series, 2001

[17] N. Ozay, U. Topcu, and R. M. Murray, "Distributed power allocation for vehicle management systems," in Proc. IEEE CDC and ECC, Orlando, FL, USA, Dec. 2011

[18] P. Derler, E. A. Lee, and A. S. Vincentelli, "Modeling cyber-physical systems," Proceedings of the IEEE, vol. 100, no. 1, pp. $13-28$, jan. 2012

[19] T. Wongpiromsarn, U. Topcu, and R. M. Murray, "Receding horizon temporal logic planning," 2010, submitted to IEEE Transactions on Automatic Control.

[20] F. Borrelli, Constrained Optimal Control of Linear and Hybrid Systems. Springer, Lecture Notes in Control and Information Sciences, vol 290, 2003.

[21] R. Bloem, B. Jobstmann, N. Piterman, A. Pnueli, and Y. Sa'ar, "Synthesis of reactive(1) designs," Journal of Computer and System Sciences, vol. 78, no. 3, pp. 911 - 938, may 2012.

[22] A. Pnueli, Y. Sa'ar, and L. D. Zuck. (2010) JTLV : A framework for developing verification algorithms. 22nd International Conference on Computer Aided Verification. [Online]. Available: http://jtlv.ysaar.net

[23] T. Wongpiromsarn, U. Topcu, N. Özay, H. Xu, and R. M. Murray, "TuLiP: a software toolbox for receding horizon temporal logic planning," in $H S C C, 2011$, software available at http://tulip-control.sf.net/ 